\title{
Mutants of a Temperature-Sensitive Two-P Domain Potassium Channel
}

\author{
Maya T. Kunkel, ${ }^{1}$ Duncan B. Johnstone, ${ }^{3}$ James H. Thomas, ${ }^{3}$ and Lawrence Salkoff ${ }^{1,2}$ \\ Departments of ${ }^{1}$ Anatomy and Neurobiology and ${ }^{2}$ Genetics, Washington University School of Medicine, St. Louis, Missouri \\ 63110, and ${ }^{3}$ Department of Genetics, University of Washington, Seattle, Washington 98195
}

Within the Caenorhabditis elegans genome there exist at least 42 genes encoding TWK (two-P domain $\mathrm{K}^{+}$) channels, potassium channel subunits that contain two pore regions and four transmembrane domains. We now report the first functional characterization of a TWK channel from $C$. elegans. Although potassium channels have been reported to be activated by a variety of factors, TWK-18 currents increase dramatically with increases in temperature. Two mutant alleles of the twk-18 gene confer uncoordinated movement and paralysis in C. elegans. Expression of wild-type and mutant TWK-18 channels in Xenopus oocytes showed that mutant channels express much larger potassium currents than wild-type channels. Promoter-green fluorescent protein fusion experiments indicate that TWK-18 is expressed in body wall muscle. Our genetic and physiological data suggest that the movement defects observed in mutant twk-18 animals may be explained by an increased activity of the mutant TWK-18 channels.

Key words: potassium channel; temperature; C. elegans; uncoordinated; mutant; TWIK; TASK; TREK; TRAAK; KCNK; unc110; mah-2
Recently, a systematic survey of predicted $\mathrm{K}^{+}$channel genes within the complete genomic sequence of Caenorhabditis elegans revealed that the majority of the many genes encoding $\mathrm{K}^{+}$channels belong to the TWK family (Salkoff and Jegla, 1995; Wei et al., 1996). Members of this $\mathrm{K}^{+}$channel family have four transmembrane domains and two $\mathrm{P}$ regions and have been designated twk (two-P domain $\mathrm{K}^{+}$channel) genes in $C$. elegans. The $\mathrm{P}$ domain is the defining element of $\mathrm{K}^{+}$channels containing a contiguous stretch of seven amino acids, TTXGYGD, flanked by two transmembrane domains. Both extensive site-directed mutagenesis and knowledge of the crystal structure of a simple $\mathrm{K}^{+}$channel have demonstrated that this region confers the high selectivity for $\mathrm{K}^{+}$to these channels (Heginbotham et al., 1994; Doyle et al., 1998). Although the P region is highly conserved, the membrane topology of metazoan $\mathrm{K}^{+}$channel subunits is more diverse; thus $\mathrm{K}^{+}$channels belong to one of three structural classes: those spanning the membrane two or six times and containing only one $\mathrm{P}$ region per subunit and those belonging to the TWK family with four transmembrane segments and two P regions per subunit. Studies from the mammalian TWK channel TWIK-1 suggest that members of this class of $\mathrm{K}^{+}$channel subunits associate as dimers in contrast to members of the other two structural classes that form as tetramers (Lesage et al., 1996b).

TWK channels have been found in mammals, Drosophila, Arabidopsis, and C. elegans (Salkoff et al., 1999). At least nine mammalian TWK channels have been identified from expressed sequence tags (ESTs), whereas a much larger number were identified through sequence analysis of the $C$. elegans genome (Lesage et al., 1996a; Wei et al., 1996; Fink et al., 1998; Reyes et al., 1998; Chavez et al., 1999; Maingret et al., 1999; Salinas et al., 1999; Salkoff et al., 1999). However, on the basis of the $\mathrm{K}^{+}$channels compiled from the

Received April 14, 2000; revised July 18, 2000; accepted July 26, 2000

This research was supported by grants to L.S. and J.H.T. from the National Institutes of Health. M.T.K. was supported by postdoctoral training grants from the McDonnell Center for Cellular and Molecular Neurobiology and the National Institutes of Health. We thank Danielle Thierry-Mieg for providing the e1913 allele and preliminary mapping data, and Mike Nonet and his laboratory for assistance in mapping the cn110 allele. We thank Dr. R. Horvitz for helpful discussions. We are grateful to members of our laboratories for helpful comments during the course of this work.

The GenBank accession number for the $t w k-18$ cDNA sequence is AF083650

Correspondence should be addressed to Dr. Lawrence Salkoff, Department of Anatomy and Neurobiology, Washington University, 660 South Euclid Avenue, Box 8108, St. Louis, MO 63110. E-mail: salkoffl@thalamus.wustl.edu.

Copyright (C) 2000 Society for Neuroscience $0270-6474 / 00 / 207517-\bullet \$ 15.00 / 0$
C. elegans genome and the rarity in which these are represented among $C$. elegans ESTs, the known mammalian TW K channels may represent only a small fraction of all the mammalian TWKs.

Previous reports on members of the TWK channel family suggest that these channels are regulated by diverse factors. TWIK-1 and TWIK-2 currents are reportedly inhibited by intracellular acidity (Lesage et al., 1996a), whereas TASK currents are inhibited by extracellular acidity (Duprat et al., 1997). Both TRAAK and TREK currents are activated by arachidonic acid and membrane stretch (Patel et al., 1998; Maingret et al., 1999). More recently, some members of the TWK channel family have been shown to be activated in response to volatile general anesthetics (Kindler et al., 1999; Patel et al., 1999).

Here we present evidence that two previously described movement-defective mutants of $C$. elegans map to the gene encoding the TWK channel subunit TWK-18. Although the functional properties of cloned TWK channels from mammals have been reported, this is the first report of the functional properties of a TWK channel from $C$. elegans. We demonstrate that the activity of this $C$. elegans TWK channel is sharply augmented by higher temperature. Such temperature sensitivity could conceivably be a mechanism for regulating membrane excitability over the broad temperature ranges that these animals may encounter. This temperature sensitivity extends the diversity by which TWK channels are known to be regulated. Last, through our analyses of the mutant channel subunits, we present a mechanism by which these mutations confer their behavioral phenotype in $C$. elegans.

\section{MATERIALS AND METHODS}

Mapping mutant alleles to twk-18. C. elegans strains were propagated as described (Brenner, 1974). twk-18(e1913) [previously designated unc110(e1913)] was mapped with respect to two cloned genes, unc-115 and egl-15. From parents of genotype twk-18(e1913sd $)+$ egl-15 $(n 484) /+u n c-$ $115(e 2225)+$, we isolated viable uncoordinated (Unc) Egl recombinants, which were either of genotype $t w k-18+e g l-15 /++e g l-15$ or $t w k-18+$ egl-15/+unc-115 egl-15. These two genotypes are phenotypically indistinguishable, which minimizes a picking bias for mapping purposes. From 112 recombinants, 50 segregated $u n c-115$ and 62 did not, placing twk-18 in a narrow region that contained the $t w k-18$ candidate $\mathrm{K}^{+}$channel subunit. Mutations were identified by PCR sequencing the $t w k-18$ coding sequence, which was PCR amplified from populations of wild-type or mutant animals. twk-18(cn110) [previously designated mah-2(cn110)] had previously been mapped on the X chromosome to the left of dpy-6. twk-18(cn110) was first mapped with respect to $d p y-7$ and unc-6. From $t w k-18(c n 110) / d p y-7$ unc-6 heterozygotes, 7 of 7 Unc, non-Dpy (unc-6+/unc-6 dpy-7) segregated twk-18(cn110) and 15 of 15 Dpy, non-Unc (+ dpy-7/unc-6 dpy-7) did not 
segregate $t w k-18(c n 110)$, suggesting that $t w k-18(c n 110)$ maps to the right of $d p y-7$. In a subsequent experiment, from $t w k-18(\mathrm{cn} 110) / d p y-6 \mathrm{egl}-15$ heterozygotes, 6 of 27 Dpy animals segregated $t w k-18(\mathrm{cn} 110)$, mapping the cn110 allele near the twk-18 locus. The genomic region encompassing $t w k-18$ was amplified by PCR from mutant $t w k-18(\mathrm{cn} 110)$ animals. Portions of this $\sim 3 \mathrm{~kb}$ PCR fragment were sequenced. Sequence results from two independent PCR reactions indicated a single-base change at residue 840 of the coding region substituting an isoleucine for a methionine at amino acid 280 .

To revert $t w k-18(g f)$, we made use of $t w k-18(s a 589)$, a partial revertant allele of $t w k-18$ that had been isolated in a previous revertant screen (Johnstone, 1999). twk-18(sa589) animals are paralyzed yet viable alleles of $t w k-18$ and thus permitted an improved screen for recessive suppressors of $t w k-18(g f)$. In this screen, $t w k-18(s a 589)$ hermaphrodites were mutagenized with methanesulfonic acid ethyl ester and screened two generations later for improved movement. From a screen of $\sim 100,000$ genomes, 66 revertants were isolated. Fifty-two of the revertants were wild type; all were tightly linked to $t w k-18$ and are presumed intragenic alleles. This was confirmed by sequencing several of these 52 revertants; one of these revertants contains an early nonsense codon within the first transmembrane segment. The remaining 14 revertants were only partially suppressed and all were extragenic. Eleven of these 14 extragenic mutations caused hyperactive locomotion and hyperactive egg laying in the absence of the twk-18(gf) mutation, phenotypes characteristic of mutations affecting the goa-1 pathway (Mendel et al., 1995). By genetic complementation analysis, three of the hyperactive alleles proved to be alleles of goa-1 (D. B. Johnstone and J. H. Thomas, unpublished observations). These goa-1 mutations likely suppress $t w k-18(g f)$ indirectly, because goa-1 is not expressed in body wall muscle (Mendel et al., 1995). We hypothesize that they suppress $t w k-18(g f)$ by indirectly increasing membrane excitability in body wall muscles. The other eight hyperactive mutations may be alleles of other genes in the goa-1 pathway and were not further analyzed. Among $>80$ revertants of $t w k-18(g f)$, we were unable to find any evidence that TWK-18 subunits require other subunits for channel formation in vivo.

Green fluorescent protein-promoter fusions. PCR segments were amplified from genomic DNA prepared from wild-type C. elegans and subcloned into pPD95.69 (Fire Lab vector kit) to generate pPD95.69-twk-18P. The amplified fragment included $2.75 \mathrm{~kb}$ of sequence upstream of the ATG and the encoded N-terminal end of TW K-18 up to alanine 30 within the second exon. Two $C$. elegans lines were generated by co-injection of pPD95.69twk-18P $(20 \mathrm{ng} / \mu \mathrm{l})$ with the dominant marker encoding rol-6 $(150 \mathrm{ng} / \mu \mathrm{l})$ (Mello et al., 1991). Two additional transformed lines were generated by coinjection of pPD95.69-twk-18P with a rescuing lin-15 construct (Huang et al., 1994) into lin-15(n765ts) animals at concentrations of 10 and 50 $\mathrm{ng} / \mu \mathrm{l}$, respectively. All four lines displayed fluorescence only in body wall muscle.

Cloning twk-18. The $5^{\prime}$ and $3^{\prime}$ ends of the coding sequence of $t w k-18$ were predicted by Genefinder (P. Green, University of Washington, Seattle, WA) to be separate adjacent genes in cosmid C24A3. However, sequence data from a C. elegans EST, yk305H4 (Y. Kohara, National Institute of Genetics, Mishima, Japan), indicated that the two separate genes together encode $t w k-18$. $t w k-18$ was cloned by PCR from first-strand cDNA using a forward primer upstream of the predicted ATG and a reverse primer downstream of the stop codon that had been identified from the EST sequence. The cDNA encoding $t w k-18$ was subcloned into the pOX vector (Wei et al., 1994) and confirmed by sequencing. Mutant cDNA constructs were created by overlap PCR using the Quikchange site-directed mutagenesis kit (Stratagene, La Jolla, CA) and confirmed by sequence analysis.

Oocyte expression and electrophysiological analysis. Xenopus oocytes were isolated and treated as described (Soreq and Seidman, 1992). In vitro capped cRNA was synthesized using the mMESSAGE mMACHINE kit (Ambion, Austin, TX). Fifty nanoliters of cRNA were injected into oocytes using standard methods at concentrations between 0.1 and $2 \mu \mathrm{g} / \mu \mathrm{l}$. In experiments comparing basal whole-cell current amplitudes for wildtype and mutant subunits, cRNA was injected at equal concentrations (1 $\mu \mathrm{g} / \mu \mathrm{l})$ into same-stage oocytes obtained from the same harvest. Injected oocytes were recorded $1 \mathrm{~d}$ after injection in ND96 (in mM: $96 \mathrm{Na}^{+}, 2 \mathrm{~K}^{+}$, $1.8 \mathrm{Ca}^{2+}, 1 \mathrm{Mg}^{2+}$, and 5 HEPES, pH 7.4) with $1 \mathrm{~mm} 4,4^{\prime}$-diisothiocyanatostilbene-2,2'-disulfonic acid (DIDS), clamped at $-80 \mathrm{mV}$, and stepped from -100 to $+40 \mathrm{mV}$ in $10 \mathrm{mV}$ increments. Data were collected from three different oocyte preparations and pooled. TWK-18(e1913) whole-cell current magnitudes were significantly larger than TWK$18(\operatorname{cn} 110)$ magnitudes $(t$ test, $p<0.001)$. Similarly, TWK-18(cn110)injected oocytes expressed significantly larger whole-cell currents than TWK-18 (wt)-injected oocytes $(p<0.001)$.

Because of the high levels of TWK-18(e1913) currents, TW K-18(e1913) was injected at lower cRNA concentrations than wild-type TWK-18 and TWK-18(cn110) in experiments examining the temperature dependence of the TWK-18 subunits. The bath temperature was controlled using a Peltier device (Cambion Corp.) and monitored using an electronic temperature sensor. For these experiments, a current elicited by a $+40 \mathrm{mV}$ step from a holding potential of $-80 \mathrm{mV}$ was recorded at temperatures from 15 to $35^{\circ} \mathrm{C}$ in $5^{\circ} \mathrm{C}$ increments. Current amplitudes in these experiments were normalized to the $25^{\circ} \mathrm{C}$ current obtained from respective oocytes. Normalized values were averaged with data obtained from multiple oocyte preparations and plotted (Sigmaplot 5.0).
Patch-clamp analysis. Borosilicate glass electrodes were pulled to resistances of 2-2.5 $\mathrm{M} \Omega$ and fire-polished. Inside-out and on-cell patches were obtained from devitellinized Xenopus oocytes expressing the designated channel subunit. Traces were acquired using an Axopatch 200A (Axon Instruments, Foster City, CA), digitized at $50 \mathrm{kHz}$, and filtered at 5 or 10 $\mathrm{kHz}$. Data were analyzed using pClamp 7 (Axon Instruments). In experiments to determine the $\mathrm{K}^{+}$selectivity of TW K-18, currents were recorded from inside-out macropatches expressing many TWK-18(e1913) channels. The pipette solution contained (in $\mathrm{mM}$ ): $155 \mathrm{~K}^{+}, 5 \mathrm{Na}^{+}, 1.8 \mathrm{Ca}^{2+}, 1$ $\mathrm{Mg}^{2+}$, and 5 HEPES, $\mathrm{pH}$ 7.4. The patch was subjected to voltage ramps from -80 to $+80 \mathrm{mV}$ and perfused with varying concentrations of $\mathrm{K}^{+}$and $\mathrm{Na}^{+}$. Salines perfused on the intracellular surface consisted of (in mM): $155 \mathrm{~K}^{+}$and $5 \mathrm{Na}^{+}, 120 \mathrm{~K}^{+}$and $40 \mathrm{Na}^{+}, 80 \mathrm{~K}^{+}$and $80 \mathrm{Na}^{+}$, or $40 \mathrm{~K}^{+}$and $120 \mathrm{Na}^{+}$

In experiments to determine single-channel conductance and mean open time, pipette and bath salines both contained (in $\mathrm{mM}$ ): $160 \mathrm{~K}^{+}, 1.8 \mathrm{Ca}^{2+}$ $1 \mathrm{Mg}^{2+}$, and 5 HEPES, $\mathrm{pH}$ 7.4. Single-channel conductances from mutant TWK-18 subunits were determined from fits of amplitude histograms using pClamp software. Because wild-type TWK-18 single-channel events were rare, the conductance was determined and averaged from multiple individual single-channel openings. Mean open times were determined from single exponential fits of dwell time histograms using pClamp software.

\section{RESULTS}

\section{Mutations in twk-18 affect $C$. elegans locomotion}

Two alleles of $t w k-18, e 1913$ and $c n 110$, were previously identified in a screen for Unc mutants and a screen for temperature-sensitive paralytic mutants, respectively. These alleles were originally described as unc-110(e1913) (Reiner et al., 1995) and mah-2(cn110) (Hosono et al., 1985), but we will hereafter refer to them as twk-18(e1913) and twk-18(cn110), in accord with the molecular designation of this gene as the TWK gene $t w k-18$ (Wei et al., 1996).

twk-18(e1913) and twk-18(cn110) are gain-of-function alleles that exhibit semidominant defects in locomotion (Hosono et al., 1985; Thomas, 1990; Reiner et al., 1995). Homozygous twk18(e1913) animals are inviable, but heterozygous twk-18(e1913)/+ animals display uncoordinated movement (Thomas, 1990; Reiner et al., 1995). The lethality of e1913 when homozygous can be explained by the severity of the resulting muscle contraction defect. A minimal level of body wall muscle contraction is required for embryonic morphogenesis in C. elegans and mutations that prevent this, arrest at a characteristic stage of development termed the two-fold stage (Williams and Waterston, 1994). From the broods of e1913/+ animals, approximately one-fourth arrest at the two-fold stage of development (Johnstone and Thomas, unpublished observations). The second allele of $t w k-18, c n 110$, was isolated in a temperature-sensitive screen for paralytic mutants. $t w k-18(\mathrm{cn} 110)$ mutants move well at lower temperatures and become paralyzed at elevated temperatures (Hosono et al., 1985).

\section{Mapping of twk-18(e1913) and twk-18(cn110)}

Earlier work showed that $t w k-18(e 1913)$ maps near the center of the $\mathrm{X}$ chromosome (Reiner et al., 1995). We mapped twk18(e1913) with respect to two cloned genes, unc-115 and egl-15, which allowed us to correlate genetic distance with a set of overlapping cosmids on the X chromosome (Fig. 1; see Materials and Methods). This region of the physical map contained a predicted $\mathrm{K}^{+}$channel subunit of the TWK family, $t w k-18$, a candidate gene for a defect in cell excitability. By completely sequencing this locus from $t w k-18(e 1913)$ animals, we identified a single missense mutation in the fourth exon of $t w k-18$. The mutation substitutes an aspartate for a conserved glycine $(\mathrm{G} \rightarrow \mathrm{D})$ at the base of the second transmembrane segment, M2 (Fig. $1 B, C$ ). The $t w k-18(c n 110)$ mutant was also identified as an allele of $t w k-18$ through mapping and sequence analysis of the $t w k-18$ locus (see Materials and Methods). cn110 is a missense mutation in the sixth exon resulting in the substitution of isoleucine for methionine $(\mathrm{M} \rightarrow \mathrm{I})$ in the loop between the second $\mathrm{P}$ domain and the fourth transmembrane segment, M4 (Fig. 1B,C).

To establish that the mutations in the $t w k$ - 18 coding sequence cause the Unc phenotype, we reverted the twk-18(e1913) gain-offunction allele (see Materials and Methods). We isolated $>60$ revertants that were tightly linked to e1913 in crosses and showed improved movement. These are presumably intragenic loss-of- 
A

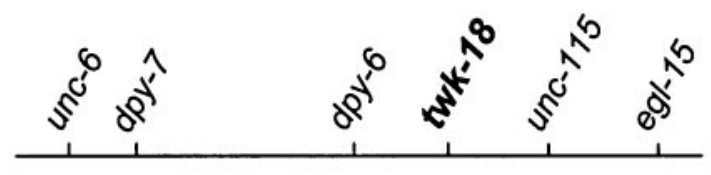

0.5 map units

B

1 MAIVAQGVSTILTTFQKTFKGLLPLIILVAYTLLGAWIFWMI EGENERE

50 MLIEQQKERDELIRRTVYKINQLQIKRQRRLMTAEEEYNRTAKVLTTFQ

99 ETLGIVPADMDKDIHWTFLGSIFYCMTVYTTIGYGNIVPGTGWGRPATI M2 e1913(G $\rightarrow D)$

148 LYAFIGIPLTVLSIYCLGSLFAKGCKMLWRFFLKSTRVVSKDLSNKISE

197 AADNIEEGTTAITPSAEKTENNDDDLLSFPISGLLLITVIWVIFCAVLF P2 $\quad \mathrm{Cn} 110(M \rightarrow I) \quad M 4$

246 TFLEEWDFGTSLYFTLISFTTIGFGDILPSDYDFMPIVGVLLLIGLSLV

295 STVMTLIJQQQIEALASGMKDNIDQEYARALNEAREDGEVDEHVDPEEDP

344 ENNKKSFDAVISRMNWSKRGLYYLLPDSQKKELAKQSEKKMGRKSIKIQ

393 TDNDLLETLIREEILKAELNNEMHKYTAPRSSHQPKLVYSDVREKEVPI

442 EVVRVEHFNHGNEDYLEHDI
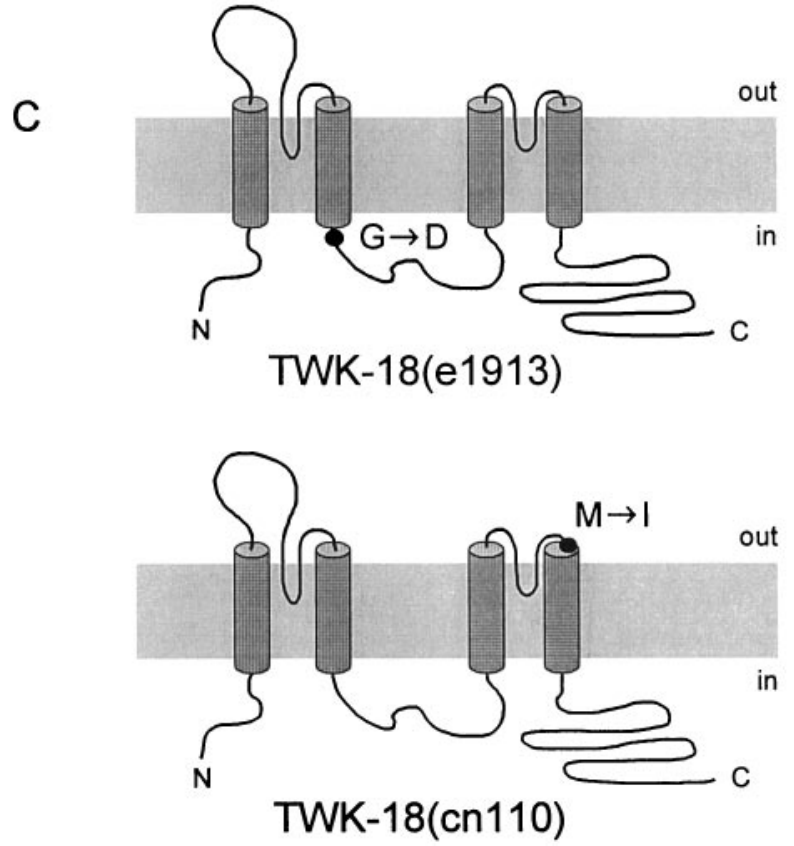

Figure 1. Identification of twk-18(e1913) and twk-18(cn110). A, Genetic map of the twk-18 gene. The center of the $\mathrm{X}$ chromosome is shown twk-18(e1913) and twk-18(cn110) were mapped to the interval between dpy-6 and unc-115. The predicted potassium channel gene, twk-18, was sequenced from both mutants, and each had a missense mutation within its coding region. $B$, Amino acid sequence of TWK-18. The four predicted transmembrane domains are boxed and labeled $M 1-M 4$. The $\mathrm{P}$ regions are underlined and labeled $P 1$ and $P 2$. The amino acid residues mutated in the twk-18 mutants are marked in bold, and the substitution is indicated for each allele. $C$, Amino acid changes identified in the mutant $t w k-18$ alleles illustrated on a diagram showing the putative topology of TWK-18 subunits. In $t w k-18(e 1913)$ animals, an aspartate residue is substituted for a highly conserved glycine at the intracellular boundary of M2 (top). In twk-18(cn110) animals, an isoleucine is substituted for a methionine residue at the extracellular boundary of M4 (bottom).
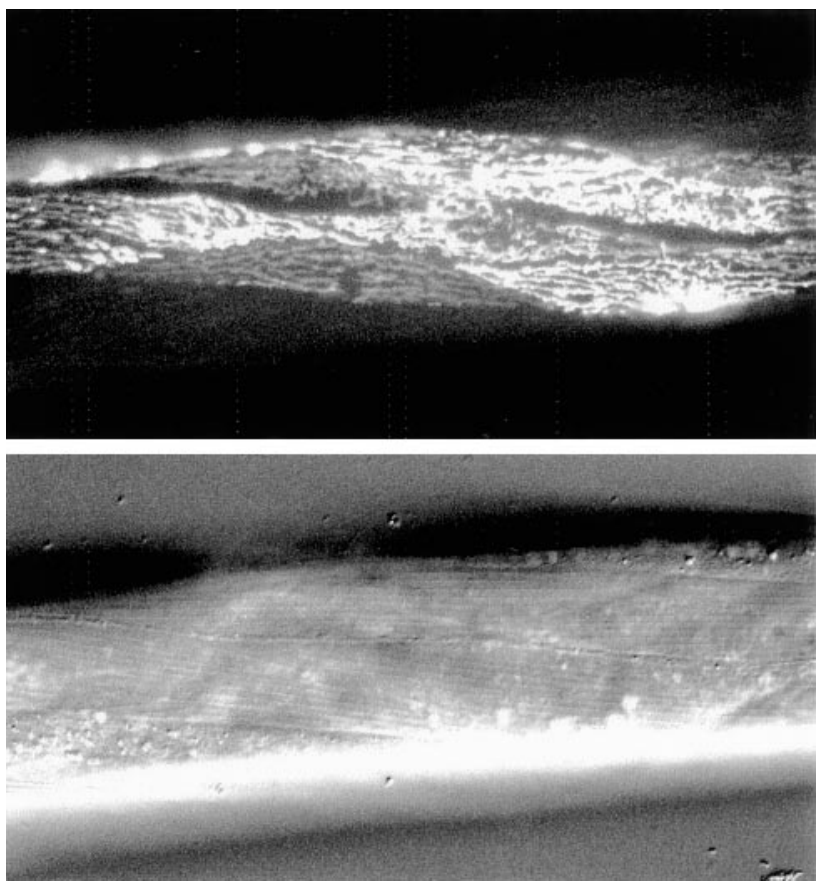

Figure 2. TWK-18 is expressed in body wall muscle. A transgenic worm is shown expressing a translational fusion of the intracellular $\mathrm{N}$ terminus of TWK-18 fused to GFP under control of the $t w k-18$ promoter. The promoter region includes $2.75 \mathrm{~kb}$ of sequence upstream from the initiator methionine. Animals were co-transformed with rol-6 DNA, identified by the Rol marker phenotype, and then screened for GFP expression. A representative transgenic animal is shown expressing the twk-18::gfp fusion in body wall muscle. Bottom panel, Nomarski image of the same animal. Four independent lines were isolated; all had GFP expression in body wall muscle.

function revertants, and their phenotype appeared grossly wildtype. Sequencing of the $t w k-18$ locus from several of these revertants confirmed the presence of the original e1913 mutation as well as a new mutation that could explain a loss of function. For example, one of these revertants resulted in a stop codon predicted to truncate TWK-18 within the first transmembrane domain. We presume that the loss of function of TWK-18 is nondetrimental because of functional redundancy (see Discussion). Thus, these screens confirmed that the gain-of-function mutations identified in twk-18 confer the Unc phenotype. Extragenic suppressors within genes necessary for TWK-18 channel formation or function could also be identified from these screens; however, we found no evidence that TWK-18 subunits require other subunits for channel formation in vivo (see Materials and Methods).

\section{TWK-18 is expressed in body wall muscle}

We examined the expression pattern of TWK-18 in vivo by designing a green fluorescent protein (GFP) promoter fusion for $C$. elegans transformation experiments. Genomic sequence including $2.75 \mathrm{~kb}$ of sequence upstream from the initiator methionine was used in a translational fusion of the second twk-18 exon to $g f p$. Four independent $t w k-18:: g f p$ lines were generated, which displayed a GFP signal only in body wall muscle (Fig. 2). The expression in body wall muscle is consistent with a defect in body wall muscle contraction.

\section{Wild-type and mutant TWK-18 subunits express outwardly rectifying $\mathrm{K}^{+}$currents}

To analyze the effect of the point substitutions on channel function, wild-type and mutant channel subunits were expressed in Xenopus oocytes and subjected to electrophysiological analysis with twoelectrode voltage-clamp and patch-clamp techniques. Recordings from oocytes injected with equal amounts of cRNAs show that wild-type TWK-18 channels expressed very small, outwardly rectifying whole-cell currents (at $40 \mathrm{mV}, 0.7 \pm 0.37 \mu \mathrm{A}$, mean $\pm \mathrm{SD}$; 

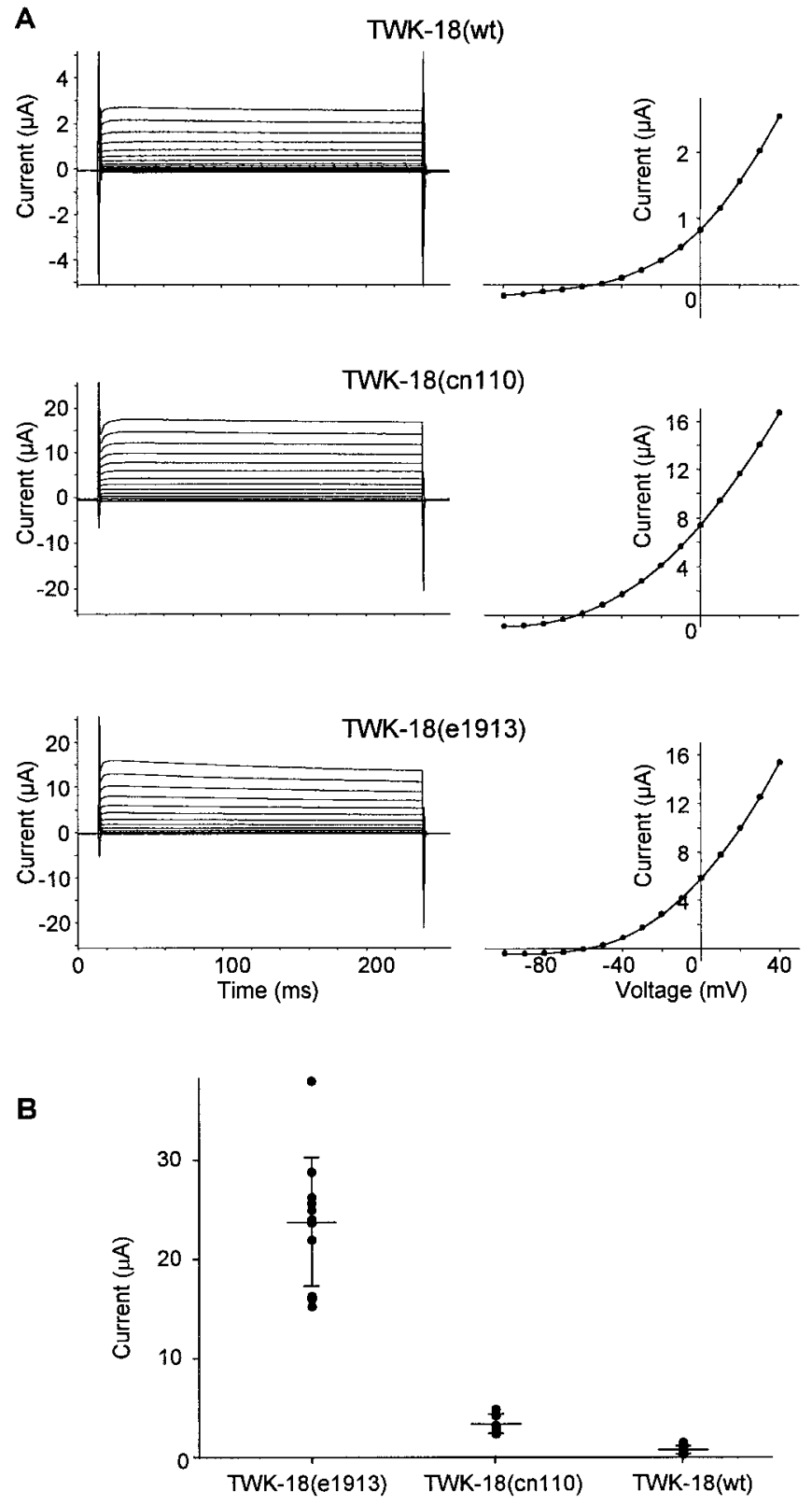

Figure 3. Wild-type and mutant TWK-18 whole-cell currents. TWK-18 channels express an outwardly rectifying current. $A$, Oocytes were injected with $50 \mathrm{nl}$ of $2 \mu \mathrm{g} / \mu \mathrm{l}$ wild-type TWK-18 or TWK-18(cn110) cRNA or 0.1 $\mu \mathrm{g} / \mu \mathrm{l}$ TWK-18(e1913) cRNA. The bath solution contained ND96 with 1 mM DIDS to inhibit endogenous chloride currents. The oocytes were recorded at $35^{\circ} \mathrm{C}$ and held at $-80 \mathrm{mV}$ and then stepped from -100 to +40 $\mathrm{mV}$ in $10 \mathrm{mV}$ increments. Current-voltage relations from the traces on the left are plotted on the right and reflect similar outward rectification for both the wild-type and mutant TWK-18 channels. $B$, Scatterplot depicting the distribution of whole-cell currents recorded from oocytes at $25^{\circ} \mathrm{C}$ that had been injected with equal amounts of cRNA $(1 \mu \mathrm{g} / \mu \mathrm{l})$ encoding wild-type $(w t ; n=12)$ and mutant $(n=11$ each) TWK-18 channels as indicated. The mean \pm SD current for TWK-18(e1913) is $23.7 \pm 6.5 \mu \mathrm{A}$; that for TW K-18(cn110) is $3.3 \pm 1.0 \mu \mathrm{A}$; and that for wild-type TWK-18 is $0.7 \pm$ $0.37 \mu \mathrm{A}$, represented by horizontal lines. The whole-cell current magnitudes between TWK-18(e1913) and TWK-18(cn110) and between TWK18(cn110) and wild type TWK-18 were statistically distinct $(p<0.001, t$ test).

$n=12)$, whereas TWK-18(e1913) and TWK-18(cn110) expressed dramatically larger currents $(23.7 \pm 6.5 \mu \mathrm{A} ; n=11$; and $3.3 \pm 1.0$ $\mu \mathrm{A} ; n=11$, respectively; (Fig. $3 B$ ). Although the magnitude of channel activity varied between the wild-type and mutant TWK-18 channels, other macroscopic properties such as the current-voltage relation and time dependence of activation appeared similar (Fig.
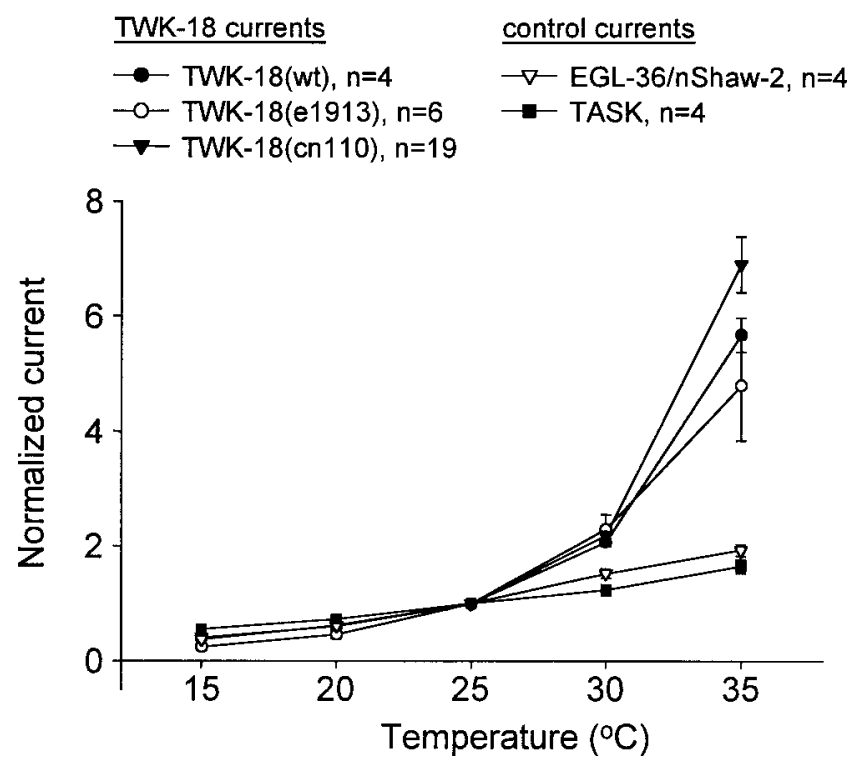

Figure 4. TWK-18 currents display steep temperature dependence. Steady-state whole-cell current amplitudes were measured at temperatures from 15 to $35^{\circ} \mathrm{C}$. Oocytes expressing wild-type (wt) and mutant TWK-18 channels were held at $-80 \mathrm{mV}$ and stepped to a test pulse of $+40 \mathrm{mV}$. Currents were normalized to the current magnitude measured at $+40 \mathrm{mV}$ at $25^{\circ} \mathrm{C}$. Because of its large currents, TWK-18(e1913) cRNA was injected at a 20 -fold dilution $(0.1 \mu \mathrm{g} / \mu \mathrm{l})$ compared with TWK-18 (wt) and TWK18 (cn110) cRNA $(2 \mu \mathrm{g} / \mu \mathrm{l})$. TW K-18 (wt) $(\bullet)$ and mutant channels TWK18(e1913) (O) and TWK-18(cn110) $(\boldsymbol{\nabla})$ display a similar nonlinear increase in channel activity with increasing temperature. Control recordings were performed using nShaw2/EGL-36, a voltage-gated delayed rectifier $\mathrm{K}^{+}$ channel $(\nabla)$, and TASK, a mammalian TW K channel (ם). Control currents were fit to a line with $r^{2}=0.99$ for EGL-36 and 0.97 for TASK, whereas TWK-18-derived currents were poorly fit $\left[r^{2}=0.76\right.$ for TWK-18 (wt), 0.72 for TWK-18(cn110), and 0.82 for TW K-18(e1913)].

$3 A$ ). For both wild-type and mutant currents, the conductancevoltage relations do not approach saturation even at $+100 \mathrm{mV}$. Consistent with this observation, we noted that single-channel activity does not approach saturation over this voltage range. This reflects the extremely low open probability of the TWK-18 channel (see below). Thus, except for the much larger currents seen in recordings from the mutant TW K-18 constructs, other macroscopic properties appear similar. These physiological studies are consistent with the behavioral phenotypes observed in mutant twk-18 animals, because an excessive $\mathrm{K}^{+}$conductance across the body wall muscle membrane would be expected to inhibit muscle excitability. Furthermore, the severity of the mutant phenotypes correlates with the relative magnitude of whole-cell currents recorded in Xenopus oocytes.

\section{TWK-18 currents display a steep dependence on temperature}

The twk-18(cn110) mutant was initially isolated on the basis of its temperature-sensitive phenotype; the animal is motile at temperatures between 15 and $22^{\circ} \mathrm{C}$ but Unc at $>22^{\circ} \mathrm{C}$ and paralyzed at $\geq 25^{\circ} \mathrm{C}$ (Hosono et al., 1985; our unpublished observations). Underlying this temperature-sensitive behavioral phenotype could be a temperature-sensitive effect specific to the TWK-18(cn110) channels themselves. Hence we examined the properties of both wildtype and mutant channels over this temperature range. Whole-cell currents were recorded from Xenopus oocytes expressing either wild-type or mutant TWK-18 channels. Currents were examined at a test pulse of $+40 \mathrm{mV}$ from a holding potential of $-80 \mathrm{mV}$, and the test pulse was repeated at $5^{\circ} \mathrm{C}$ temperature increments from 15 to $35^{\circ} \mathrm{C}$ (Fig. 4). To standardize the vastly different current amplitudes for wild-type and mutant channels, the amplitudes were normalized to currents obtained for each channel type at $25^{\circ} \mathrm{C}$ (Fig. 4). The results demonstrate that all three channels, TWK-18 (wt), TWK-18(e1913), and TWK-18(cn110), show a similar steep, 
temperature-dependent increase over this temperature range (Fig. 4). Thus, the unusual temperature-dependent increase in current amplitude is a property of wild-type as well as mutant channels and not the result of a mutational effect on the thermostability of the TWK-18(cn110) channel.

The temperature dependence of TWK-18 current amplitude is unusually high. As a control, we examined the temperature dependence of current amplitude for a mammalian member of the TWK family, TASK (Duprat et al., 1997), and a voltage-dependent channel from C. elegans, EGL-36/nShaw-2 (Johnstone et al., 1997). The relation between the current amplitude and temperature was similar for both of these channels with a Q10 ( $\Delta$ current amplitude with $\Delta 10^{\circ} \mathrm{C}$ ) of 2 over the range of $15-35^{\circ} \mathrm{C}$ (Fig. 4). This is in marked contrast to the dramatic increase observed with TWK-18 wild-type and mutant subunits. Over the temperature range examined, the TW K-18 channels showed a Q10 of $\sim 2-3$ at the lower temperature $\left(15^{\circ} \mathrm{C}\right)$ that increased to 6 at higher temperatures $\left(30^{\circ} \mathrm{C}\right)$. This nonlinear response to temperature suggests that the TWK-18 channel may function as a temperature-gated $\mathrm{K}^{+}$channel in vivo. This degree of temperature dependence is similar to that seen for the capsaicin receptor, a nonspecific cation channel that is believed to be the sensor for noxiously high temperatures (Caterina et al., 1997). However, major distinguishing features of the TWK-18 channels are that they are sensitive at much lower temperatures and that they are selective for $\mathrm{K}^{+}$over sodium or calcium. These distinguishing features may reflect the different physiological roles of these channels; the function of the capsaicin receptor is to depolarize the cell to trigger an excitable response, whereas one function of the TWK-18 channel may be to inhibit cell excitability in response to increasing temperatures.

\section{Single-channel recordings}

Activity from inside-out patches containing either mutant or wildtype TWK-18 channels was recorded to characterize basic singlechannel properties. In addition to revealing single-channel properties, it was hoped that these studies would reveal the differences in whole-cell current amplitude observed between wild-type and mutant channels and the mechanism of outward rectification. Observations of single-channel activity were made in both the cellattached and inside-out patch configurations. The channel was observed in these patches as a distinctive high-conductance channel with a very short mean open time. The primary difference seen between patches containing wild-type and mutant channels was the number of single-channel events observed; events were much more frequent in patches containing mutant channel subunits.

\section{Single-channel conductances and mean open times}

Single-channel conductances were measured in inside-out patch recordings with equimolar $\mathrm{K}^{+}(160 \mathrm{~mm})$ in the bath and pipette. The values observed were $182 \pm 21 \mathrm{pS}$ for TWK-18(e1913), $164 \pm$ $48 \mathrm{pS}$ for TWK-18(cn110), and $130 \pm 18 \mathrm{pS}$ for wild-type TWK-18. Conductances for mutant channels were calculated from amplitude histograms. However, because openings of wild-type channels are rare, their conductance measurements were estimated by measuring the amplitudes of individually selected single-channel events and may underestimate the unitary conductance. The small differences seen in the single-channel conductances among mutant and wild-type channels may be attributable to the amino acid changes present near the mouth of the channel or to experimental difficulties inherent in measuring such brief events. Importantly, these small differences cannot account for the up to 30-fold differences seen between the whole-cell current amplitudes of wild-type and mutant channels.

The single-channel mean open time was determined through single exponential fits of the dwell time distribution from patch recordings at $+60 \mathrm{mV}$. The average calculated value obtained from data from at least three independent patches was $0.21 \pm 0.02 \mathrm{msec}$ for TWK-18(e1913), $0.23 \pm 0.04 \mathrm{msec}$ for TWK-18( $\mathrm{cn} 110)$, and $0.19 \pm 0.07 \mathrm{msec}$ for wild-type TWK-18. Thus the mean open time does not differ significantly between wild-type and mutant channels.
Because neither single-channel conductance nor mean channel open time can account for the larger whole-cell current amplitudes seen with the mutant channels, the larger current amplitudes might be attributable to an increased frequency of channel openings. However, because of the very low single-channel mean open time (averaging $0.2 \mathrm{msec}$ ) and very low open channel probability, the number of TWK-18 channels in a patch cannot be determined; thus the open channel probability for single channels cannot be accurately measured.

\section{Mechanism of outward rectification}

TWK-18 currents observed in whole-cell current recordings from Xenopus oocytes exhibit an apparent outward rectification. Because these channels lack the classic S4 voltage-sensing region of voltagedependent $\mathrm{K}^{+}$channels, outward rectification must be accounted for by other factors. To observe the single-channel behavior that might correspond to this rectification, we examined single-channel openings at voltages from -60 to $+60 \mathrm{mV}$. Single-channel openings were observed at both positive and negative voltages; however, at the negative voltages, the mean channel open time was significantly decreased. Because of the extremely short openings at negative voltages, accurate measurement of mean open time was difficult. However, a preliminary estimate of the mean open time in the inward direction indicates a value of far $<100 \mu \mathrm{sec}$ (relative to $200 \mu \mathrm{sec}$ in the outward direction). A representative example of this difference in single-channel behavior at +60 and $-60 \mathrm{mV}$ is shown in Figure $5 A$. This difference in single-channel behavior at different voltages is likely to be responsible for at least a part of the outward rectification observed for whole-cell currents. The mechanism responsible for this single-channel behavior has not been determined. Although we observed that this change in gating character is most marked in crossing the $\mathrm{K}^{+}$equilibrium potential, it does not apparently depend on the presence of a divalent ion acting as an open channel blocker, because similar channel openings were observed in the absence of either magnesium or calcium ions (M. T. Kunkel and L. Salkoff, unpublished observations). Notably, this difference in gating is not lost in inside-out patch recordings, indicating that the difference in mean open time at positive and negative potentials is not attributable to a diffusible intracellular factor but may be a property intrinsic to the channel.

\section{Potassium selectivity}

To determine the ion selectivity of the TWK-18 channel, recordings were made from inside-out macropatches expressing multiple TWK-18(e1913) channels. The pipette solution contained (in mM): $155 \mathrm{~K}^{+}$and $5 \mathrm{Na}^{+}$. The patch was subjected to voltage ramps from -80 to $+80 \mathrm{mV}$ and the intracellular (cytoplasmic) surface exposed to salines containing four different concentrations of $\mathrm{K}^{+}$and $\mathrm{Na}^{+}$(in mM: $155 \mathrm{~K}^{+}$and $5 \mathrm{mM} \mathrm{Na}^{+}, 120 \mathrm{~K}^{+}$and $40 \mathrm{Na}^{+}, 80 \mathrm{~K}^{+}$ and $80 \mathrm{Na}^{+}$, and $40 \mathrm{~K}^{+}$and $120 \mathrm{Na}^{+}$). The reversal potentials under these four conditions were determined by observing the voltage at the zero current level during the ramp. Under all four conditions, the reversal potential $\left(\mathrm{P}_{\mathrm{R}}\right)$ of the channel closely approximated the calculated $\mathrm{K}^{+}$equilibrium potential $\left(\mathrm{E}_{\mathrm{K}}\right)$. The $\mathrm{P}_{\mathrm{R}}$ values compared with $\mathrm{E}_{\mathrm{K}}$ were, respectively, 1.5-0.0, 7.3-6.4, 14.316.7 , and $24.9-34 \mathrm{mV}$. Figure $5 B$ plots these data and demonstrates that the reversal potential of the channel is predominantly influenced by the equilibrium potential for $\mathrm{K}^{+}$. These experiments demonstrate a high selectivity for $\mathrm{K}^{+}$over $\mathrm{Na}^{+}$for the TWK-18 channel.

\section{DISCUSSION}

twk-18 is one of at least 42 TWK genes in the C. elegans genome that are predicted to encode $\mathrm{K}^{+}$channel subunits containing two $\mathrm{P}$ regions and four transmembrane domains. TWK-18 channels express an outwardly rectifying $\mathrm{K}^{+}$current, and the properties of rectification may significantly depend on the fact that singlechannel open time differs for inward and outward current. TWK-18 currents dramatically increase in response to temperature and are thus "temperature-gated." The two gain-of-function missense al- 
A
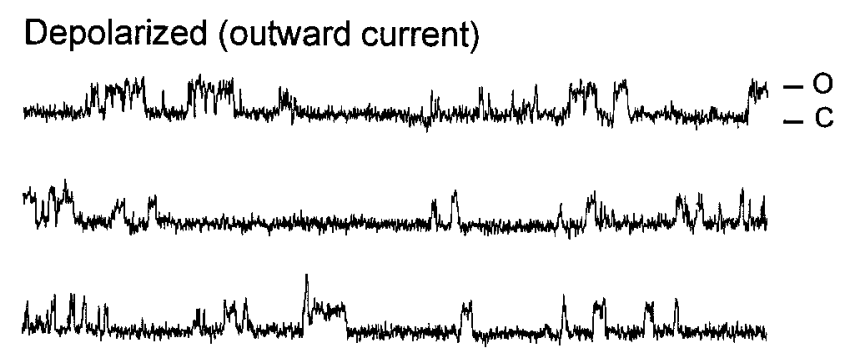

\section{Hyperpolarized (inward current)}
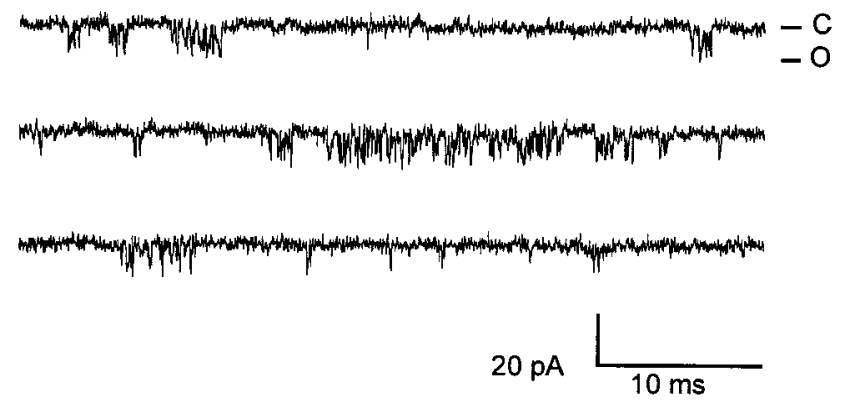

B
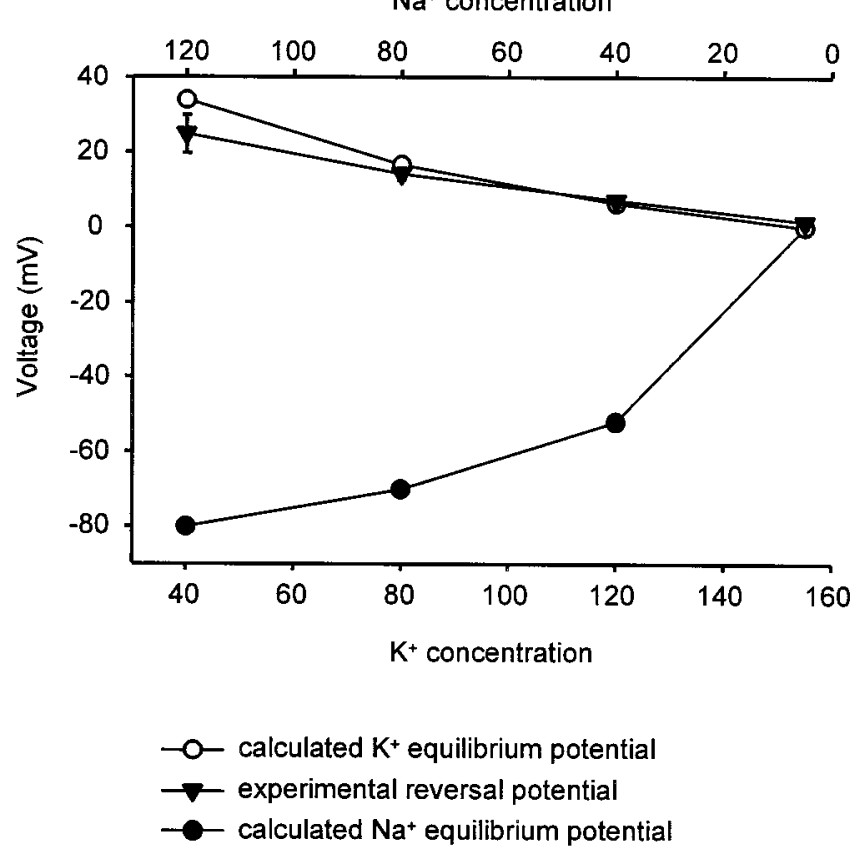

Figure 5. TWK-18(e1913) single-channel currents. $A$, Inside-out patch recording obtained at room temperature from an oocyte expressing TWK18(e1913) channels. The bath and pipette solutions contained symmetrical $\mathrm{K}^{+}(160 \mathrm{mM})$. The patch was depolarized to $+60 \mathrm{mV}(t o p)$ or hyperpolarized to $-60 \mathrm{mV}$ (bottom). $C$, Zero current levels. Channel openings $(O)$ are observed at both depolarized and hyperpolarized potentials, but openings at hyperpolarized potentials are much briefer than openings at depolarized potentials. $B$, TW K-18 is highly selective for $\mathrm{K}^{+}$. Voltage ramps from -80 to $+80 \mathrm{mV}$ were applied to a patch containing TWK-18(e1913) channels. The pipette contained (in $\mathrm{mm}$ ): $155 \mathrm{~K}^{+}$and $5 \mathrm{Na}^{+}$. The intracellular face was exposed to salines containing different concentrations of $\mathrm{K}^{+}$and $\mathrm{Na}^{+}$ (in mM: $155 \mathrm{~K}^{+}$and $5 \mathrm{Na}^{+}, 120 \mathrm{~K}^{+}$and $40 \mathrm{Na}^{+}, 80 \mathrm{~K}^{+}$and $80 \mathrm{Na}^{+}$, and $40 \mathrm{~K}^{+}$and $120 \mathrm{Na}^{+}$). The plot demonstrates that the experimental reversal potential $(\boldsymbol{\nabla})$ closely followed the predicted $\mathrm{K}^{+}$equilibrium potential $(O)$ but not the $\mathrm{Na}^{+}$equilibrium potential $(\mathbf{O})$. This demonstrates high selectivity for $\mathrm{K}^{+}$over $\mathrm{Na}^{+}$. leles of $t w k-18$ confer a semidominant, flaccidly paralyzed phenotype in C. elegans, which is likely to be attributable to the fact that mutant channels express much larger outward $\mathrm{K}^{+}$currents than wild-type channels. Expression of GFP driven by the $t w k-18$ promoter indicates expression limited to body wall muscle. We conclude that elevated outward $\mathrm{K}^{+}$currents in body wall muscle inhibit muscle excitation necessary to drive locomotion.

Several $t w k$ family cDNAs have been cloned from mammals and heterologously expressed in Xenopus oocytes or cell culture. The currents are regulated by a variety of factors, including $\mathrm{pH}$, lipid metabolites, membrane stretch, and volatile anesthetics. Our preliminary data indicated that TWK-18 currents are gated by $\mathrm{pH}$ but not by membrane stretch (Kunkel and Salkoff, unpublished observations); however, here in this report we demonstrate that TWK-18 activity is sensitive to temperature. The nonlinear increase in TWK-18 currents between 15 and $35^{\circ} \mathrm{C}$ suggests that TWK-18 channels undergo a temperature-induced conformational change that might be expected of a channel designed to be unusually sensitive to temperature. In contrast to TWK-18, currents from the mammalian TWK channel TASK showed a linear response to temperature, maintaining a Q10 of $\sim 2$ over the entire temperature range $\left(15-35^{\circ} \mathrm{C}\right)$. There is one other report of a cloned temperature-sensitive channel, the capsaicin receptor (Caterina et al., 1997). This nonselective cation channel is activated by capsaicin but is also activated by high temperatures $\left(55^{\circ} \mathrm{C}\right)$, thereby signaling noxious temperature stimuli to the organism. Does the temperature dependence of TWK-18 have any biological significance in $C$. elegans? Wild-type TWK-18 currents may contribute only a small fraction to the total $\mathrm{K}^{+}$conductance of the membrane at $15^{\circ} \mathrm{C}$, but because of the nonlinear increase in current with increasing temperature, this fractional contribution may rise. Perhaps this offers a kind of thermostat and negative feedback control over muscle membrane excitability as temperatures rise. Nevertheless, TWK-18 channels do not appear to be essential for normal gross muscle function over this temperature range under laboratory conditions, because null $t w k$ - 18 alleles appear nominally wild-type. The lack of a phenotype from null alleles may be attributable to a functional redundancy supplied by other TWK family channels expressed in body wall muscle. In addition to $t w k-18$ there are at least three other TWK family members expressed in the body wall muscle of C. elegans (A. Butler, G. Paz-y-Mino C., and L. Salkoff, unpublished observations). Why there are four or more TWK family channel genes expressed in body wall muscle and whether they represent independent current-carrying systems or form heteromultimers remain to be determined.

\section{Larger currents expressed by mutant channels}

Neither single-channel conductance nor mean channel open time was found to make a significant contribution to the larger wholecell currents seen with mutant channels. Thus, larger currents expressed by mutant channels are likely to be caused by either an increased frequency of channel openings or a larger number of channels at the plasma membrane. The locations of the $t w k$ $18(e 1913)$ and $t w k-18(\mathrm{cn} 110)$ mutations in the second and fourth transmembrane domains (M2 and M4) are consistent with mutations that might be expected to change the gating characteristics of $\mathrm{K}^{+}$channels. The M1-P-M2 and M3-P-M4 regions of TWK channels are analogous to the S5-P-S6 regions of voltage-gated $\mathrm{K}^{+}$ channels. Several $\mathrm{K}^{+}$channel subunits in $C$. elegans have been cloned recently on the basis of semidominant, activating alleles, and in all but one instance, the subunits had mutations in S6 (Johnstone et al., 1997; Elkes et al., 1997; Davis et al., 1999; Weinshenker et al., 1999; D. J. Reiner and J. H. Thomas, unpublished observations). Furthermore, random mutagenesis of the yeast two-P domain $\mathrm{K}^{+}$channel TOK1 (Ykc1) similarly revealed the importance of this region in $\mathrm{K}^{+}$channel gating (Loukin et al., 1997). In another study, analysis of the S6 region in Shaker with cysteine-linking reagents drew similar conclusions with greater detail, suggesting that the S6 segment forms an "activation gate" at the inner vestibule of $\mathrm{K}^{+}$channels (Liu et al., 1997). In addition, an 
investigation by Perozo et al. (1999) using site-directed spinlabeling methods and electron paramagnetic resonance spectroscopy on the Streptomyces KcsA channel further illustrates the importance of the base of the second transmembrane segment in channel gating. The implication from these studies is that the transmembrane segment immediately after the pore domain (S6 in Shaker channels and M2 or M4 in TWK channels) forms part of a gate at the inner vestibule of the channel, and mutations in these transmembrane segments may affect the energetics of transitions between closed and open states of the channel. On the other hand, there is no evidence that these TWK channels actually "gate" in the sense of changing conformational states. One of the TWK channels in a mammalian system was termed an "open rectifier" (Leonoudakis et al., 1998) to connote that the channel was actually ungated and usually in an open state. Thus, the apparent openings and closings observed during single-channel analyses could be the consequence of a blocking and deblocking process by an unidentified domain of the channel or an as yet unknown extrinsic factor.

An alternative explanation to account for the larger whole-cell currents seen with the mutant channels could be that the $t w k-18$ gain-of-function mutations facilitate more efficient protein processing or an increased stability of the subunit within the plasma membrane. For example, a mutation in a cytoplasmic domain that signals retention of the protein in the endoplasmic reticulum (ER), such as the RKR motif present in Kir6.1/2 (Zerangue et al., 1999), would facilitate passage of functional channels through the ER. Indeed, the mammalian KCNK6 TWK channel, which fails to

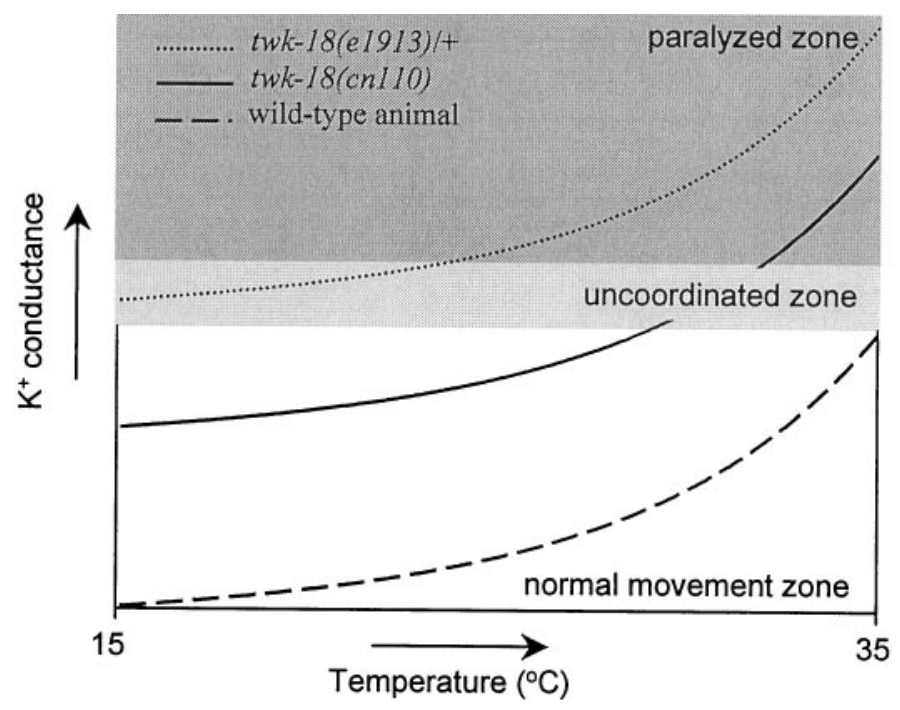

Figure 6. Relationship among temperature, current amplitudes, and movement. Illustrated is the relationship between the temperature-dependent levels of $\mathrm{K}^{+}$conductance in body wall muscle and the movement defects seen in twk-18 mutants. Three zones are suggested, relating temperature and the amount of $\mathrm{K}^{+}$conductance in body wall muscle to the movement ability of the animal. In the normal movement zone shown at the bottom, the amount of $\mathrm{K}^{+}$conductance never impedes the movement ability of the animal. In the uncoordinated zone lightly shaded in the middle, the amount of $\mathrm{K}^{+}$conductance is such that movement is impaired but not eliminated. In the paralyzed zone darkly shaded at the top, the amount of $\mathrm{K}^{+}$conductance is so great that all movement is inhibited. The idealized plots for wild-type and mutant animals reflect the nonlinear increase in $\mathrm{K}^{+}$conductance with increasing temperature. Although the $\mathrm{K}^{+}$conductance in wildtype animals increases with increasing temperature (bottom trace), the absolute amount of conductance never reaches a level that impedes the movement of the animal. Thus, wild-type animals remain in the normal movement zone at all temperatures. In contrast, the conductance in $t w k$ 18(cn110) animals (middle trace) begins in the normal movement zone but with increasing temperature enters the uncoordinated zone, and with further elevation of temperature, the amount of $\mathrm{K}^{+}$conductance enters the paralyzed zone. Consequently, twk-18(cn110) animals exhibit a temperature-dependent, uncoordinated phenotype. In the most severely affected mutant animals, twk-18(e1913)/+ (top trace), the amount of $\mathrm{K}^{+}$ conductance is in the uncoordinated zone at room temperature; a further rise of temperature increases $\mathrm{K}^{+}$conductance into the paralyzed zone. express currents, is believed to be retained in the ER and thus may possess such a motif (Salinas et al., 1999). However, the mutations in $t w k-18$ seem to be in an unusual position for such an effect. Another possibility is that the $t w k-18$ gain-of-function mutations might relieve the need for other types of protein interactions required for channel function. These alternative possibilities were not addressed in our experiments.

\section{Temperature-sensitive phenotype of twk-18(cn110) mutants}

The temperature-sensitive behavioral phenotype seen with $t w k$ 18(cn110) mutants may be a result of a temperature-induced increase in $\mathrm{K}^{+}$conductance above a critical level. Both mutant and wild-type currents show similar temperature sensitivity profiles, so the question arises of why the $t w k-18(e 1913)$ mutant is Unc at all temperatures, whereas the mutant twk-18(cn110) shows a temperature-conditional behavioral phenotype (wild-type behavior is unaffected over the same temperature range). Expression of $\mathrm{K}^{+}$ currents in Xenopus oocytes shows that the magnitude of the whole-cell current from both mutant channels is much larger than that observed from the wild-type channel, but the magnitude differs greatly between them [TWK-18(e1913) > TWK-18(cn110) > TWK-18 (wt)]. Thus, the starting level of muscle membrane $\mathrm{K}^{+}$ conductance is apparently much greater in the twk-18(e1913) mutant than in the $t w k-18(c n 110)$ mutant. Presumably this level in the twk-18(e1913) mutant exceeds a threshold that confers a severe Unc phenotype, and this is true even at the lowest temperatures studied. In the $t w k-18(c n 110)$ mutant, however, the level of muscle membrane $\mathrm{K}^{+}$conductance at the lower temperatures is apparently below the critical threshold that confers an Unc phenotype. As the temperature is raised, the temperature-induced increase in $\mathrm{K}^{+}$conductance surpasses the critical level, thus producing the temperature-sensitive phenotype seen with the $t w k-18(c n 110) \mathrm{mu}-$ tant. In contrast, the activity of the wild-type channel is low enough so that the critical level of $\mathrm{K}^{+}$conductance is not reached even at $35^{\circ} \mathrm{C}$. This mechanism of temperature-conditional paralysis is illustrated in Figure 6.

Interesting questions now remain to be investigated with respect to TWK-18 properties and function. Through patch-clamp analysis of TWK-18 at different temperatures, one may be able to determine the mechanism by which ion channels can be gated by temperature. That is, temperature may increase channel activity by increasing the channel mean open time, conductance, or frequency of channel openings. It is intriguing that multiple TWK channels are expressed in $C$. elegans body wall muscle. Our data suggest that TWK-18 can function as a homomeric channel, but the coexpression of multiple TWK channels in the same tissue might suggest that two subunits could form a heteromeric channel with novel properties. Finally, the significance of the sensitivity of TWK-18 to temperature and its role in tuning and modulating cellular excitability in vivo remain to be determined.

\section{REFERENCES}

Brenner S (1974) The genetics of Caenorhabditis elegans. Genetics 77:71-94.

Caterina MJ, Schumacher MA, Tominaga M, Rosen TA, Levine JD, Julius D (1997) The capsaicin receptor: a heat-activated ion channel in the pain pathway. Nature 389:816-824.

Chavez RA, Gray AT, Zhao BB, Kindler CH, Mazurek MJ, Mehta Y, Forsayeth JR, Yost CS (1999) TWIK-2, a new weak inward rectifying member of the tandem pore domain potassium channel family. J Biol Chem 274:7887-7892.

Davis MW, Fleischhauer R, Dent JA, Joho RH, Avery L (1999) A mutation in the $C$. elegans EXP-2 potassium channel that alters feeding behavior. Science 286:2501-2504.

Doyle DA, Morais Cabral J, Pfuetzner RA, Kuo A, Gulbis JM, Cohen SL, Chait BT, MacKinnon R (1998) The structure of the potassium channel: molecular basis of K+ conduction and selectivity. Science 280:69-77.

Duprat F, Lesage F, Fink M, Reyes R, Heurteaux C, Lazdunski M (1997) TASK, a human background $\mathrm{K}^{+}$channel to sense external $\mathrm{pH}$ variations near physiological pH. EMBO J 16:5464-5471.

Elkes DA, Cardozo DL, Madison J, Kaplan JM (1997) EGL-36 Shaw channels regulate $C$. elegans egg-laying muscle activity. Neuron 19:165-174. 
Fink M, Lesage F, Duprat F, Heurteaux C, Reyes R, Fosset M, Lazdunski M (1998) A neuronal two $\mathrm{P}$ domain $\mathrm{K}^{+}$channel stimulated by arachidonic acid and polyunsaturated fatty acids. EMBO J 17:3297-3308.

Heginbotham L, Lu Z, Abramson T, MacKinnon R (1994) Mutations in the $\mathrm{K}+$ channel signature sequence. Biophys J 66:1061-1067.

Hosono R, Kuno S, Midsukami M (1985) Temperature-sensitive mutations causing reversible paralysis in Caenorhabditis elegans. Exp Zool 235:409-421.

Huang LS, Tzou P, Sternberg PW (1994) The lin-15 locus encodes two negative regulators of Caenorhabditis elegans vulval development. Mol Biol Cell 5:395-411.

Johnstone D (1999) Genetic analysis of potassium channels in C. elegans. $\mathrm{PhD}$ dissertation, University of Washington.

Johnstone DB, Wei A, Butler A, Salkoff L, Thomas JH (1997) Behavioral defects in C. elegans egl-36 mutants result from potassium channels shifted in voltage-dependence of activation. Neuron 19:151-164.

Kindler CH, Yost CS, Gray AT (1999) Local anesthetic inhibition of baseline potassium channels with two pore domains in tandem. Anesthesiology 90:1092-1102.

Leonoudakis D, Gray AT, Winegar BD, Kindler CH, Harada M, Taylor DM, Chavez RA, Forsayeth JR, Yost CS (1998) An open rectifier potassium channel with two pore domains in tandem cloned from rat cerebellum. J Neurosci 18:868-877.

Lesage F, Guillemare E, Fink M, Duprat F, Lazdunski M, Romey G, Barhanin J (1996a) TWIK-1, a ubiquitous human weakly inward rectifying $\mathrm{K}^{+}$channel with a novel structure. EMBO J 15:1004-1011.

Lesage F, Reyes R, Fink M, Duprat F, Guillemare E, Lazdunski M (1996b) Dimerization of TWIK-1 K ${ }^{+}$channel subunits via a disulfide bridge. EMBO J 15:6400-6407.

Liu Y, Holmgren M, Jurman ME, Yellen G (1997) Gated access to the pore of a voltage-dependent $\mathrm{K}^{+}$channel. Neuron 19:175-184.

Loukin SH, Vaillant B, Zhou X-L, Spalding EP, Kung C, Saimi Y (1997) Random mutagenesis reveals a region important for gating fo the yeast $\mathrm{K}^{+}$channel Ykc1. EMBO J 16:4817-4825.

Maingret F, Fosset M, Lesage F, Lazdunski M, Honore E (1999) TRAAK is a mammalian neuronal mechano-gated $\mathrm{K}^{+}$channel. J Biol Chem 274:1381-1387.

Mello CC, Kramer JM, Stinchcomb D, Ambros V (1991) Efficient gene transfer in C. elegans: extrachromosomal maintenance and integration of transforming sequences. EMBO J 10:3959-3970.

Mendel JE, Korswagen HC, Liu KS, Hajdu-Cronin YM, Simon MI, Plasterk RH, Sternberg PW (1995) Participation of the protein Go in multiple aspects of behavior in C. elegans. Science 167:1652-1655.
Patel AJ, Honore E, Maingret F, Lesage F, Fink M, Duprat F, Lazdunski M (1998) A mammalian two pore domain mechano-gated S-like K+ channel. EMBO J 17:4283-4290.

Patel AJ, Honore E, Lesage F, Fink M, Romey G, Lazdunski M (1999) Inhalational anesthetics activate two-pore-domain background $\mathrm{K}+$ channels. Nat Neurosci 2:422-426.

Perozo E, Cortes DM, Cuello LG (1999) Structural rearrangements underlying $\mathrm{K}^{+}$-channel activation gating. Science 285:73-78.

Reiner DJ, Weinshenker D, Thomas JH (1995) Analysis of dominant mutations affecting muscle excitation in Caenorhabditis elegans. Genetics 141:961-976.

Reyes R, Duprat F, Lesage F, Fink M, Salinas M, Farman N, Lazdunski M (1998) Cloning and expression of a novel $\mathrm{pH}$-sensitive two pore domain $\mathrm{K}^{+}$channel from human kidney. J Biol Chem 273:30863-30869.

Salinas M, Reyes R, Lesage F, Fosset M, Heurteaux C, Romey G, Lazdunski M (1999) Cloning of a new mouse two-P domain channel subunit and a human homologue with a unique pore structure. J Biol Chem 274:11751-11760.

Salkoff L, Jegla T (1995) Surfing the DNA databases for K+ channels nets yet more diversity. Neuron 15:489-492.

Salkoff L, Kunkel MT, Wang Z-W, Butler A, Nonet M, Wei A (1999) The impact of the $C$. elegans genome sequencing project on $\mathrm{K}^{+}$channel biology. In: Potassium ion channels: molecular structure, and diseases (Kurachi Y, Jan LY, Lazdunski M, eds), pp 9-27. San Diego: Academic.

Soreq H, Seidman S (1992) Xenopus oocyte microinjection: from gene to protein. Methods Enzymol 207:225-265.

Thomas JH (1990) Genetic analysis of defecation in Caenorhabditis elegans. Genetics 124:395-399.

Wei A, Solaro C, Lingle C, Salkoff L (1994) Calcium sensitivity of BKtype $\mathrm{KCa}$ channels determined by a separable domain. Neuron 13:671-681.

Wei A, Jegla T, Salkoff L (1996) Eight potassium channel families revealed by the $C$. elegans genome project. Neuropharmacology $35: 805-829$.

Weinshenker D, Wei A, Salkoff L, Thomas JH (1999) Block of an ethera-go-go-like $\mathrm{K}^{+}$channel by imipramine rescues egl-2 excitation defects in Caenorhabditis elegans. J Neurosci 19:9831-9840.

Williams BD, Waterston RH (1994) Genes critical for muscle development and function in Caenorhabditis elegans identified through lethal mutations. J Cell Biol 124:475-490.

Zerangue N, Schwappach B, Jan YN, Jan LY (1999) A new ER trafficking signal regulates the subunit stoichiometry of plasma membrane K(ATP) channels. Neuron 22:537-548. 